\title{
A Variance-Constrained Approach to Recursive State Estimation for Time-Varying Complex Networks with Missing Measurements *
}

\author{
Jun $\mathrm{Hu}^{\mathrm{a}}$, Zidong Wang ${ }^{\mathrm{b}}$, Steven Liu ${ }^{\mathrm{c}}$, Huijun Gao ${ }^{\mathrm{d}}$ \\ ${ }^{a}$ Department of Applied Mathematics, Harbin University of Science and Technology, Harbin 150080, China. \\ ${ }^{\mathrm{b}}$ Department of Computer Science, Brunel University London, Uxbridge, Middlesex, UB8 3PH, U.K. \\ ${ }^{\mathrm{c}}$ Department of Electrical and Computer Engineering, University of Kaiserslautern, Kaiserslautern 67663, Germany. \\ ${ }^{\mathrm{d}}$ Research Institute of Intelligent Control and Systems, Harbin Institute of Technology, Harbin 150001, China.
}

\begin{abstract}
In this paper, the recursive state estimation problem is investigated for an array of discrete time-varying coupled stochastic complex networks with missing measurements. A set of random variables satisfying certain probabilistic distributions is introduced to characterize the phenomenon of the missing measurements, where each sensor can have individual missing probability. The Taylor series expansion is employed to deal with the nonlinearities and the high-order terms of the linearization errors are estimated. The purpose of the addressed state estimation problem is to design a time-varying state estimator such that, in the presence of the missing measurements and the random disturbances, an upper bound of the estimation error covariance can be guaranteed and the explicit expression of the estimator parameters is given. By using the Riccatilike difference equations approach, the estimator parameter is characterized by the solutions to two Riccati-like difference equations. It is shown that the obtained upper bound is minimized by the designed estimator parameters and the proposed state estimation algorithm is of a recursive form suitable for online computation. Finally, an illustrative example is provided to demonstrate the feasibility and effectiveness of the developed state estimation scheme.
\end{abstract}

Key words: State estimation; Time-varying complex networks; Variance constraints; Missing measurements; Recursive approach; Riccati-like difference equations.

\section{Introduction}

Complex networks are composed of a group of interconnected nodes under certain topological structures. As is well known, the scale-free networks and small-world networks are two popular classes of complex networks characterized by the power-law degree distributions and the short path lengths as well as high clustering $[1,23]$. Noting the importance and popularity of the complex networks, increasing research attention has been devoted to various aspects of the network structure $[14,15,26]$. During the past decade, the dynamics analysis of the complex networks has become a very active topic of research due to its application potentials in a wide range of realworld networks such as biological networks, computer networks, electrical power grids, cyber-physical systems, social networks and technological networks. In particular, considerable effort has been made towards the sta-

* This work was supported in part by the National Natural Science Foundation of China under Grants 61329301, $61273156,61333012,11301118$ and 11271103 , the Youth Science Foundation of Heilongjiang Province of China under Grant QC2015085, the China Postdoctoral Science Foundation under Grants 2015T80482 and 2014M560376, Jiangsu Planned Projects for Postdoctoral Research Funds under Grant 1402004A, the Royal Society of the U.K., and the Alexander von Humboldt Foundation of Germany. Corresponding author Jun $\mathrm{Hu}$.

Email addresses: hujun2013@gmail.com (Jun Hu), Zidong.Wang@brunel.ac.uk (Zidong Wang). bility, synchronization, impulsive control, pinning control and state estimation problems for complex networks $[20-22,26,28,29]$.

In reality, the system states are not always available due probably to physical constraints, technological restrictions or expensive cost for measuring $[5,7,12]$. Therefore, the state estimation problem has recently received tremendous research interest $[2,3,9]$. To better understand the complex networks and achieve certain engineering requirements, it is also of great significance to estimate the states of the network nodes by using the available measurements. Accordingly, a rich body of research results has been published concerning the state estimation problems for complex networks [17]. It is worth mentioning that most of the existing research concerning the state estimation problems has been carried out for time-invariant complex networks. In practice, however, almost all real-time systems evolve in a time-varying manner nature particularly when the signals are transmitted digitally. Recently, the dynamical behavior analysis of the time-varying complex networks has begun to receive some initial research attention. In [21], one of the first few attempts has been made to deal with the $H_{\infty}$ synchronization and state estimation problems for an array of discrete time-varying coupled stochastic complex networks, where the recursive linear matrix inequality approach has been employed to derive the synchronization criteria and solve the state estimation problem.

In many state estimation problems in practical engineering such as the tracking of maneuvering targets through 
a collaborative sensor network (a special complex network), the performance requirements are naturally expressed as the upper bounds on the error variances of the state estimation. In fact, for complex networks with additive stochastic disturbances, the error variance is a vitally important criterion for evaluating the estimation performances, and it seems natural for us to expect some research results on state estimation problems for complex networks with error variance (either minimized or constrained) as a performance index. To our surprise, a thorough literature review has revealed that the relevant results are very few if not none. In fact, most available dynamics analysis results for complex network$\mathrm{s}$ have been concerned with the synchronization and/or consensus behaviors that are closely related to the stability issues, and the corresponding performance indices (e.g. variance and $H_{\infty}$ constraints) have not gained adequate research attention due probably to the fact that the complicated network coupling/topology phenomena have posed significant challenges to the desired performance analysis. Nevertheless, it is of practical significance to tackle the open problem of minimum-variance state estimation for complex networks under stochastic disturbances.

For the state estimation problem of complex network$\mathrm{s}$, the available measurement information is utilized to estimate the states of the network nodes. However, due to the complexity of large-scale networks, the measurement signals may be missing/fading during the network transmission resulting from various causes such as sensors aging, intermittent sensor failures, limited bandwidth, network congestion or accidental loss of some collected data $[6,8,16,18,19]$. As such, in order to improve the estimation performance, it is vitally important to take the phenomenon of the missing measurements into account when designing the state estimator especially in the network settings $[11,13,24]$. In the past decade, the state estimation problems with missing measurements have drawn considerable research interest and a huge amount of results have been reported. To mention a few, the state estimator has been designed in [17] for complex networks with missing measurements and timevarying delays. The state estimation problem has been addressed in [22] for complex networks with sensor saturations, signal quantization and missing measurements in a unified framework. It should be pointed out that, among the reported results regarding the state estimation with missing measurements, the Bernoulli distribution is commonly employed to characterize the case where the measurements are successfully transmitted or completely missing. Nevertheless, such way of modeling missing measurements has certain limitations in practice as it fails to describe the case where only partial information is missing or fading in a networked environment [24]. To the best of the authors' knowledge, the recursive state estimation problem for time-varying stochastic complex networks with missing measurements has not been fully investigated, and the purpose of this paper is to shorten such a gap.

Motivated by the above discussions, we aim to deal with the recursive state estimation problem for an array of discrete time-varying coupled stochastic complex networks with missing measurements and random disturbances. The phenomenon of the missing measurements is char- acterized by introducing a set of random variables satisfying any discrete probabilistic distribution over the specific interval with known conditional probability. In particular, the occurrence probability of the missing measurements can be estimated via statistical tests. Through available output measurements, the missing probabilities are introduced to compensate the effects from the missing measurements when designing the time-varying state estimator for the addressed complex networks. The main contribution of this paper can be summarized as follows: 1) the variance constraint is, for the first time, introduced to cope with the state estimation problem for time-varying complex networks; 2) a compensation scheme is proposed to handle the effect from the missing measurements to the estimation performance; 3 ) the explicit form of the state estimator parameter is given based on the solution to Riccati-like difference equation$\mathrm{s}$; and 4) the developed state estimation algorithm is of a recursive form suitable for online applications.

\section{Problem Formulation and Preliminaries}

Consider the following array of discrete time-varying stochastic complex networks consisting of $N$ coupled nodes of the form:

$$
\begin{aligned}
x_{i, k+1} & =f\left(x_{i, k}\right)+\sum_{j=1}^{N} \omega_{i j} \Gamma x_{j, k}+B_{i, k} \varpi_{i, k}, \\
y_{i, k} & =\lambda_{i, k} C_{i, k} x_{i, k}+\nu_{i, k}, \quad i=1,2, \ldots, N,
\end{aligned}
$$

where $x_{i, k} \in \mathbb{R}^{n}$ is the state vector of the $i$-th node, the nonlinear function $f(\cdot)$ is known and continuously differentiable, $y_{i, k} \in \mathbb{R}^{m}$ is the measurement output from the $i$-th node of the complex network, $\varpi_{i, k}$ is the additive noise with zero-mean and covariance $Q_{i, k}>0, \nu_{i, k}$ is the additive noise with zero-mean and covariance $R_{i, k}>0$. The initial state $x_{i, 0}$ has the mean $\bar{x}_{i, 0}$. The random variables $\lambda_{i, k} \in \mathbb{R}$, which characterize the probabilistic missing phenomena, have the probability mass functions $p_{i, k}(s)$ on the intervals $\left[\alpha_{i, k}, \beta_{i, k}\right]\left(0 \leq \alpha_{i, k} \leq \beta_{i, k} \leq 1\right)$ with mathematical expectations $\mu_{i, k}$ and variances $\sigma_{i, k}^{2}$ $(i=1,2, \ldots, m)$. Also, $\lambda_{i, k}$ are $m$ uncorrelated random variables in $i$ and $k$ and are uncorrelated with other noise signals. Moreover, assume that $\lambda_{i, k}$ and all noises are confined in a same probability space. $B_{i, k}$ and $C_{i, k}$ are known and bounded matrices with appropriate dimensions. $\Gamma=\operatorname{diag}\left\{\gamma_{1}, \gamma_{2}, \ldots, \gamma_{n}\right\}$ is a matrix linking the $j$-th state variable if $\gamma_{j} \neq 0$, and $W=\left(\omega_{i j}\right) \in \mathbb{R}^{N \times N}$ is the coupling configuration matrix of the network with $\omega_{i j} \geq 0(i \neq j)$ but not all zero.

Remark 1 In the network (1)-(2), the network states are coupled through the linking matrix $\Gamma$ and the configuration matrix $W$. Such a state-connected model does have its own merit in analyzing dynamical behaviors of certain complex networks (e.g. genetic regulatory networks). $N$ evertheless, in many engineering systems such as multiagent systems and networked control system, the subsystems are often coupled/connected through their outputs and, in this case, the output-connected models can better preserve the large-scale properties of the network$s$ (e.g. the sparsity). It would be interesting to examine the impact from the output connections on the estimation performance of the complex networks in the near future. 
In this paper, we construct the following state estimator:

$$
\begin{aligned}
\hat{x}_{i, k+1 \mid k}= & f\left(\hat{x}_{i, k \mid k}\right)+\sum_{j=1}^{N} \omega_{i j} \Gamma \hat{x}_{j, k \mid k}, \\
\hat{x}_{i, k+1 \mid k+1}= & \hat{x}_{i, k+1 \mid k}+K_{i, k+1}\left(y_{i, k+1}-\mu_{i, k+1} C_{i, k+1}\right. \\
& \left.\times \hat{x}_{i, k+1 \mid k}\right),
\end{aligned}
$$

where $\hat{x}_{i, k \mid k}(i=1,2, \ldots, N)$ is the estimate of $x_{i, k}$ at time $k$ with $\hat{x}_{i, 0 \mid 0}=\bar{x}_{i, 0}, \hat{x}_{i, k+1 \mid k}$ is the one-step prediction at time $k, y_{i, k+1}$ is the actual measurement output from the $i$-th node of the complex network which is available for the estimator, $\mu_{i, k+1}$ is the available mathematical expectation of random variable $\lambda_{i, k+1}$, and $K_{i, k+1}$ is the estimator parameter to be determined.

Remark 2 For the addressed time-varying complex networks, the measurement outputs may be contaminated with the data missing and measurement noises and it is difficult to obtain the ideal output. Hence, for each subsystem, we have constructed the state estimator as in (3)-(4) by employing the actual subsystem measurement output $y_{i, k+1}$ available for the estimator. Also, the coupled configuration information of the network has been taken into consideration and the estimation information of its neighbors has been employed in (3). Moreover, the probability information of the missing measurements has been used in (4). It is expected to compensate the influence from the missing measurements onto the estimation performance so as to improve the estimation performance of the developed estimation algorithm. In this sense, the proposed estimator has certain robustness against the randomly occurred missing measurements. On the other hand, the estimation would become easier and probably more accurate if a digital processing unit could be developed for online analysis for imperfect data (e.g. randomly missing measurements) of large dimension, and this deserves further investigation.

For node $i$, let the one-step prediction error be $\tilde{x}_{i, k+1 \mid k}=$ $x_{i, k+1}-\hat{x}_{i, k+1 \mid k}$ and the estimation error be $\tilde{x}_{i, k+1 \mid k+1}=$ $x_{i, k+1}-\hat{x}_{i, k+1 \mid k+1}$. Then, we have

$$
\begin{aligned}
\tilde{x}_{i, k+1 \mid k}= & f\left(x_{i, k}\right)-f\left(\hat{x}_{i, k \mid k}\right)+\sum_{j=1}^{N} \omega_{i j} \Gamma\left(x_{j, k}-\hat{x}_{j, k \mid k}\right) \\
& +B_{i, k} \varpi_{i, k} .
\end{aligned}
$$

By using the Taylor series expansion around $\hat{x}_{i, k \mid k}$, we linearize $f\left(x_{i, k}\right)$ as follows:

$f\left(x_{i, k}\right)=f\left(\hat{x}_{i, k \mid k}\right)+A_{i, k} \tilde{x}_{i, k \mid k}+o\left(\left|\tilde{x}_{i, k \mid k}\right|\right)$,

where $A_{i, k}=\left.\frac{\partial f\left(x_{i, k}\right)}{\partial x_{i, k}}\right|_{x_{i, k}=\hat{x}_{i, k \mid k}}$ and $o\left(\left|\tilde{x}_{i, k \mid k}\right|\right)$ represents the high-order terms of the Taylor series expansion. For presentation convenience, following $[4,27]$, the high-order terms are transformed into the following formulation:

$o\left(\left|\tilde{x}_{i, k \mid k}\right|\right)=L_{i, k} \aleph_{i, k} \tilde{x}_{i, k \mid k}$

where $L_{i, k}$ are problem-dependent scaling matrices, and $\aleph_{i, k}$ are unknown time-varying matrices accounting for the linearization errors of the dynamical model that satisfy $\aleph_{i, k} \aleph_{i, k}^{T} \leq I$. Then, it follows from (5)-(7) that

$$
\begin{aligned}
\tilde{x}_{i, k+1 \mid k}= & \left(A_{i, k}+L_{i, k} \aleph_{i, k}\right) \tilde{x}_{i, k \mid k}+B_{i, k} \varpi_{i, k} \\
& +\sum_{j=1}^{N} \omega_{i j} \Gamma\left(x_{j, k}-\hat{x}_{j, k \mid k}\right) .
\end{aligned}
$$

On the other hand, according to (2) and (4), we have

$$
\begin{aligned}
& \tilde{x}_{i, k+1 \mid k+1} \\
= & \tilde{x}_{i, k+1 \mid k}-K_{i, k+1}\left(y_{i, k+1}-\mu_{i, k+1} C_{i, k+1} \hat{x}_{i, k+1 \mid k}\right) \\
= & \left(I-\mu_{i, k+1} K_{i, k+1} C_{i, k+1}\right) \tilde{x}_{i, k+1 \mid k}-K_{i, k+1} \nu_{i, k+1} \\
& -\left(\lambda_{i, k+1}-\mu_{i, k+1}\right) K_{i, k+1} C_{i, k+1} x_{i, k+1} .
\end{aligned}
$$

For the purpose of simplicity, set

$$
\begin{aligned}
& x_{k}=\left[\begin{array}{llll}
x_{1, k}^{T} & x_{2, k}^{T} & \ldots & x_{N, k}^{T}
\end{array}\right]^{T}, \\
& \varpi_{k}=\left[\begin{array}{llll}
\varpi_{1, k}^{T} & \varpi_{2, k}^{T} & \ldots & \varpi_{N, k}^{T}
\end{array}\right]^{T}, \\
& \nu_{k}=\left[\begin{array}{llll}
\nu_{1, k}^{T} & \nu_{2, k}^{T} & \ldots & \nu_{N, k}^{T}
\end{array}\right]^{T}, \\
& \hat{x}_{k+1 \mid k}=\left[\begin{array}{lllll}
\hat{x}_{1, k+1 \mid k}^{T} & \hat{x}_{2, k+1 \mid k}^{T} & \cdots & \hat{x}_{N, k+1 \mid k}^{T}
\end{array}\right]^{T}, \\
& \hat{x}_{k+1 \mid k+1}=\left[\begin{array}{llll}
\hat{x}_{1, k+1 \mid k+1}^{T} & \hat{x}_{2, k+1 \mid k+1}^{T} & \cdots & \hat{x}_{N, k+1 \mid k+1}^{T}
\end{array}\right]^{T} \text {, } \\
& \tilde{x}_{k+1 \mid k}=\left[\begin{array}{lllll}
\tilde{x}_{1, k+1 \mid k}^{T} & \tilde{x}_{2, k+1 \mid k}^{T} & \cdots & \tilde{x}_{N, k+1 \mid k}^{T}
\end{array}\right]^{T}, \\
& \tilde{x}_{k+1 \mid k+1}=\left[\begin{array}{llll}
\tilde{x}_{1, k+1 \mid k+1}^{T} & \tilde{x}_{2, k+1 \mid k+1}^{T} & \cdots & \tilde{x}_{N, k+1 \mid k+1}^{T}
\end{array}\right]^{T} \text {, } \\
& A_{k}=\operatorname{diag}\left\{A_{1, k}, A_{2, k}, \ldots, A_{N, k}\right\} \text {, } \\
& L_{k}=\operatorname{diag}\left\{L_{1, k}, L_{2, k}, \ldots, L_{N, k}\right\} \text {, } \\
& \aleph_{k}=\operatorname{diag}\left\{\aleph_{1, k}, \aleph_{2, k}, \ldots, \aleph_{N, k}\right\} \text {, } \\
& K_{k}=\operatorname{diag}\left\{K_{1, k}, K_{2, k}, \ldots, K_{N, k}\right\} \text {, } \\
& B_{k}=\operatorname{diag}\left\{B_{1, k}, B_{2, k}, \ldots, B_{N, k}\right\} \text {, } \\
& C_{k}=\operatorname{diag}\left\{C_{1, k}, C_{2, k}, \ldots, C_{N, k}\right\} \text {, } \\
& \mathbb{I}=\operatorname{diag}\{I, I, \ldots, I\}, \\
& \bar{\Lambda}_{k}=\operatorname{diag}\left\{\mu_{1, k} I, \mu_{2, k} I, \ldots, \mu_{N, k} I\right\}, \\
& \Lambda_{k}=\operatorname{diag}\left\{\lambda_{1, k} I, \lambda_{2, k} I, \ldots, \lambda_{N, k} I\right\} .
\end{aligned}
$$

Then, it follows from the above notations that we can obtain the one-step prediction error and the estimation error as $\tilde{x}_{k+1 \mid k}=x_{k+1}-\hat{x}_{k+1 \mid k}$ and $\tilde{x}_{k+1 \mid k+1}=$ $x_{k+1}-\hat{x}_{k+1 \mid k+1}$ respectively. Subsequently, by using the Kronecker product, the one-step prediction error and the estimation error can be rewritten in the following compact form:

$$
\begin{aligned}
\tilde{x}_{k+1 \mid k}= & \mathscr{A}_{k} \tilde{x}_{k \mid k}+(W \otimes \Gamma) \tilde{x}_{k \mid k}+B_{k} \varpi_{k} \\
\tilde{x}_{k+1 \mid k+1}= & \left(\mathbb{I}-K_{k+1} \bar{\Lambda}_{k+1} C_{k+1}\right) \tilde{x}_{k+1 \mid k}-K_{k+1} \\
& \times\left(\Lambda_{k+1}-\bar{\Lambda}_{k+1}\right) C_{k+1} x_{k+1}-K_{k+1} \nu_{k+1}
\end{aligned}
$$

where $\mathscr{A}_{k}=A_{k}+L_{k} \aleph_{k}$ and $\otimes$ represents the Kronecker product.

Now, according to the definition of the estimation error, let us denote the estimation error covariance as 
$P_{k+1 \mid k+1}=\mathbb{E}\left\{\tilde{x}_{k+1 \mid k+1} \tilde{x}_{k+1 \mid k+1}^{T}\right\}$ at the $k+1$-th time step. The objective of this paper is twofold. First, we aim to design an estimator of form (3)-(4) such that an upper bound of the estimation error covariance is guaranteed, i.e., we are looking for a sequence of positivedefinite matrices $\Xi_{k+1 \mid k+1}$ satisfying

$P_{k+1 \mid k+1} \leq \Xi_{k+1 \mid k+1}$.

Second, such an upper bound $\Xi_{k+1 \mid k+1}$ is minimized by properly designing the estimator parameter at each sampling instant.

Before ending this section, we recall the following lemmas which will be used in the subsequent developments.

Lemma 1 [10] Let $A=\left[a_{i j}\right]_{n \times n}$ be a real-valued matrix and $B=\operatorname{diag}\left\{b_{1}, b_{2}, \ldots, b_{n}\right\}$ be a diagonal stochastic matrix. Then

$\mathbb{E}\left\{B A B^{T}\right\}=\left[\begin{array}{cccc}\mathbb{E}\left\{b_{1}^{2}\right\} & \mathbb{E}\left\{b_{1} b_{2}\right\} & \cdots & \mathbb{E}\left\{b_{1} b_{n}\right\} \\ \mathbb{E}\left\{b_{2} b_{1}\right\} & \mathbb{E}\left\{b_{2}^{2}\right\} & \cdots & \mathbb{E}\left\{b_{2} b_{n}\right\} \\ \vdots & \vdots & \ddots & \vdots \\ \mathbb{E}\left\{b_{n} b_{1}\right\} & \mathbb{E}\left\{b_{n} b_{2}\right\} & \cdots & \mathbb{E}\left\{b_{n}^{2}\right\}\end{array}\right] \circ A$

where $\circ$ is the Hadamard product with this product being defined as $[A \circ B]_{i j}=A_{i j} \cdot B_{i j}$.

Lemma 2 [25] Given matrices $A, H, E$ and $F$ with appropriate dimensions such that $F F^{T} \leq I$. Let $X$ be a symmetric positive definite matrix and $\gamma$ be an arbitrary positive constant such that $\gamma^{-1} I-E X E^{T}>0$. Then the following inequality holds

$$
\begin{aligned}
& (A+H F E) X(A+H F E)^{T} \\
\leq & A\left(X^{-1}-\gamma E^{T} E\right)^{-1} A^{T}+\gamma^{-1} H H^{T} .
\end{aligned}
$$

Lemma 3 For matrices $M, N, X$ and $P$ with appropriate dimensions, the following equations hold

$$
\begin{aligned}
& \frac{\partial \operatorname{tr}(M X N)}{\partial X}=M^{T} N^{T}, \quad \frac{\partial \operatorname{tr}\left(M X^{T} N\right)}{\partial X}=N M, \\
& \frac{\partial \operatorname{tr}\left[(M X N) P(M X N)^{T}\right]}{\partial X}=2 M^{T} M X N P N^{T} .
\end{aligned}
$$

\section{$3 \quad$ Main Results}

In this section, according to (10)-(11), the one-step prediction error covariance and the estimation error covariance are firstly calculated. Subsequently, an upper bound of the estimation error covariance is obtained and the estimator parameter is designed to minimize such an upper bound.

Theorem 1 The one-step prediction error covariance $P_{k+1 \mid k}=\mathbb{E}\left\{\tilde{x}_{k+1 \mid k} \tilde{x}_{k+1 \mid k}^{T}\right\}$ has the following recursion:

$$
\begin{aligned}
P_{k+1 \mid k}= & {\left[A_{k}+W \otimes \Gamma+L_{k} \aleph_{k}\right] P_{k \mid k} } \\
& \times\left[A_{k}+W \otimes \Gamma+L_{k} \aleph_{k}\right]^{T}+B_{k} Q_{k} B_{k}^{T},
\end{aligned}
$$

where $Q_{k}=\operatorname{diag}\left\{Q_{1, k}, Q_{2, k}, \cdots, Q_{N, k}\right\}$.

Proof: Note that (14) follows from (10) directly, hence the proof is omitted here for brevity.
Theorem 2 The recursion of the estimation error covariance $P_{k+1 \mid k+1}$ is given as follows:

$$
\begin{aligned}
& P_{k+1 \mid k+1} \\
= & \left(\mathbb{I}-K_{k+1} \bar{\Lambda}_{k+1} C_{k+1}\right) P_{k+1 \mid k}\left(\mathbb{I}-K_{k+1} \bar{\Lambda}_{k+1} C_{k+1}\right)^{T} \\
& +K_{k+1}\left[\check{\Lambda}_{k+1} \circ\left(C_{k+1} \mathbb{E}\left\{x_{k+1} x_{k+1}^{T}\right\} C_{k+1}^{T}\right)\right] K_{k+1}^{T} \\
& +K_{k+1} R_{k+1} K_{k+1}^{T}
\end{aligned}
$$

with

$\check{\Lambda}_{k+1}=\operatorname{diag}\left\{\sigma_{1, k+1}^{2} I, \sigma_{2, k+1}^{2} I, \ldots, \sigma_{N, k+1}^{2} I\right\}$,
$R_{k+1}=\operatorname{diag}\left\{R_{1, k+1}, R_{2, k+1}, \cdots, R_{N, k+1}\right\}$.

Proof: Based on (11), we have

$$
\begin{aligned}
& P_{k+1 \mid k+1} \\
= & \left(\mathbb{I}-K_{k+1} \bar{\Lambda}_{k+1} C_{k+1}\right) P_{k+1 \mid k}\left(\mathbb{I}-K_{k+1} \bar{\Lambda}_{k+1} C_{k+1}\right)^{T} \\
& +K_{k+1} \mathbb{E}\left\{\left(\Lambda_{k+1}-\bar{\Lambda}_{k+1}\right) C_{k+1} x_{k+1} x_{k+1}^{T} C_{k+1}^{T}\right. \\
& \left.\times\left(\Lambda_{k+1}-\bar{\Lambda}_{k+1}\right)\right\} K_{k+1}^{T}+K_{k+1} R_{k+1} K_{k+1}^{T} .
\end{aligned}
$$

Subsequently, set $\tilde{\Lambda}_{k+1}=\Lambda_{k+1}-\bar{\Lambda}_{k+1}$. Then, it follows from the Lemma 1 and the property of the conditional expectation that

$$
\begin{aligned}
& \mathbb{E}\left\{\tilde{\Lambda}_{k+1} C_{k+1} x_{k+1} x_{k+1}^{T} C_{k+1}^{T} \tilde{\Lambda}_{k+1}\right\} \\
= & \mathbb{E}\left\{\mathbb{E}\left\{\tilde{\Lambda}_{k+1} C_{k+1} x_{k+1} x_{k+1}^{T} C_{k+1}^{T} \tilde{\Lambda}_{k+1}\right\} \mid \Lambda_{k+1}\right\} \\
= & \mathbb{E}\left\{\tilde{\Lambda}_{k+1} C_{k+1} \mathbb{E}\left\{x_{k+1} x_{k+1}^{T}\right\} C_{k+1}^{T} \tilde{\Lambda}_{k+1}\right\} \\
= & \check{\Lambda}_{k+1} \circ\left(C_{k+1} \mathbb{E}\left\{x_{k+1} x_{k+1}^{T}\right\} C_{k+1}^{T}\right),
\end{aligned}
$$

where $\check{\Lambda}_{k+1}$ is defined in (16). Then, we can see that (15) holds by taking (17) and (18) into account. The proof of this Theorem is complete.

Remark 3 So far, we have derived the one-step prediction error covariance and the estimation error covariance. However, there are some unknown terms $\aleph_{k}$ and $\mathbb{E}\left\{x_{k+1} x_{k+1}^{T}\right\}$ in (14) and (15) due to the consideration of the linearization errors and the missing measurements. Hence, it constitutes the difficulties on the calculation of the exact value of the estimation error covariance. In the sequel, an alternative way is employed to find an upper bound of the estimation error covariance and then minimize such an upper bound by properly designing the estimator parameters at each sampling instant.

Theorem 3 Consider the one-step prediction error covariance $P_{k+1 \mid k}$ and the estimation error covariance $P_{k+1 \mid k+1}$ in (14)-(15). Let $\epsilon_{k+1}$ and $\gamma_{k}$ be positive scalars. If the following two Riccati-like difference equations

$$
\begin{aligned}
\Xi_{k+1 \mid k}= & \left(A_{k}+W \otimes \Gamma\right)\left(\Xi_{k \mid k}^{-1}-\gamma_{k} I\right)^{-1}\left(A_{k}+W \otimes \Gamma\right)^{T} \\
& +\gamma_{k}^{-1} L_{k} L_{k}^{T}+B_{k} Q_{k} B_{k}^{T}
\end{aligned}
$$

and 


$$
\begin{aligned}
& \Xi_{k+1 \mid k+1} \\
= & \left(\mathbb{I}-K_{k+1} \bar{\Lambda}_{k+1} C_{k+1}\right) \Xi_{k+1 \mid k}\left(\mathbb{I}-K_{k+1} \bar{\Lambda}_{k+1} C_{k+1}\right)^{T} \\
& +K_{k+1}\left[\check{\Lambda}_{k+1} \circ\left(C_{k+1} \Omega_{k+1} C_{k+1}^{T}\right)\right] K_{k+1}^{T} \\
& +K_{k+1} R_{k+1} K_{k+1}^{T}
\end{aligned}
$$

with initial condition $P_{0 \mid 0} \leq \Xi_{0 \mid 0}$ have the positivedefinite solutions $\Xi_{k+1 \mid k}$ and $\Xi_{k+1 \mid k+1}$ such that the inequality $\Xi_{k \mid k}<\gamma_{k}^{-1} I$ holds for all $k \geq 0$, then the matrix $\Xi_{k+1 \mid k+1}$ is an upper bound of $P_{k+1 \mid k+1}$, i.e.,

$P_{k+1 \mid k+1} \leq \Xi_{k+1 \mid k+1}$.

Moreover, by using the following estimator parameter

$K_{k+1}=\operatorname{diag}\left\{K_{1, k+1}, K_{2, k+1}, \ldots, K_{N, k+1}\right\}$,

where

$$
\begin{aligned}
& K_{i, k+1}=\Phi_{1, i} \Xi_{k+1 \mid k} C_{k+1}^{T} \bar{\Lambda}_{k+1}^{T} \Phi_{2, i}^{T}\left(\Phi_{2, i} S_{k+1} \Phi_{2, i}^{T}\right)^{-1}, \\
& \Phi_{1, i}=\left[\begin{array}{llllll}
\underbrace{\begin{array}{lllll}
0 & \cdots & \cdots & 0
\end{array}}_{i-1} I_{n \times n} \underbrace{\begin{array}{llll}
\underbrace{}_{0} & 0 & \cdots & 0
\end{array}}_{N-i}
\end{array}\right], \\
& \Phi_{2, i}=\left[\begin{array}{llll}
\underbrace{\begin{array}{llll}
0 & \cdots & \cdots
\end{array}}_{i-1} I_{m \times m} & \underbrace{\begin{array}{llll}
0 & 0 & \cdots & 0
\end{array}}_{N-i}
\end{array}\right], \\
& \Omega_{k+1}=\left(1+\epsilon_{k+1}\right) \Xi_{k+1 \mid k}+\left(1+\epsilon_{k+1}^{-1}\right) \hat{x}_{k+1 \mid k} \hat{x}_{k+1 \mid k}^{T}, \\
& S_{k+1}=\bar{\Lambda}_{k+1} C_{k+1} \Xi_{k+1 \mid k} C_{k+1}^{T} \bar{\Lambda}_{k+1}^{T}+R_{k+1} \\
& +\check{\Lambda}_{k+1} \circ\left(C_{k+1} \Omega_{k+1} C_{k+1}^{T}\right) \text {, }
\end{aligned}
$$

the trace of the upper bound $\Xi_{k+1 \mid k+1}$ can be minimized.

Proof: We use the mathematical induction to prove this theorem. Considering the initial condition, we have $P_{0 \mid 0} \leq \Xi_{0 \mid 0}$. Assuming $P_{k \mid k} \leq \Xi_{k \mid k}$, we need to show that $P_{k+1 \mid k+1} \leq \Xi_{k+1 \mid k+1}$.

Firstly, it follows from Lemma 2 that

$$
\begin{aligned}
P_{k+1 \mid k} \leq & \left(A_{k}+W \otimes \Gamma\right)\left(P_{k \mid k}^{-1}-\gamma_{k} I\right)^{-1}\left(A_{k}+W \otimes \Gamma\right)^{T} \\
& +\gamma_{k}^{-1} L_{k} L_{k}^{T}+B_{k} Q_{k} B_{k}^{T} \\
\leq & \left(A_{k}+W \otimes \Gamma\right)\left(\Xi_{k \mid k}^{-1}-\gamma_{k} I\right)^{-1}\left(A_{k}+W \otimes \Gamma\right)^{T} \\
& +\gamma_{k}^{-1} L_{k} L_{k}^{T}+B_{k} Q_{k} B_{k}^{T} \\
= & \Xi_{k+1 \mid k},
\end{aligned}
$$

where $\gamma_{k}$ is a positive scalar. Then, we can conclude that $P_{k+1 \mid k} \leq \Xi_{k+1 \mid k}$.

Subsequently, let us handle the second term of the righthand side of (15). By using the elementary inequality

$$
\begin{array}{r}
\left(\epsilon_{k+1}^{\frac{1}{2}} \tilde{x}_{k+1 \mid k}-\epsilon_{k+1}^{\frac{1}{2}} \hat{x}_{k+1 \mid k}\right)\left(\epsilon_{k+1}^{\frac{1}{2}} \tilde{x}_{k+1 \mid k}-\epsilon_{k+1}^{\frac{1}{2}} \hat{x}_{k+1 \mid k}\right)^{T} \\
\geq 0,
\end{array}
$$

we have

$$
\begin{aligned}
& \tilde{x}_{k+1 \mid k} \hat{x}_{k+1 \mid k}^{T}+\hat{x}_{k+1 \mid k} \tilde{x}_{k+1 \mid k}^{T} \\
\leq & \epsilon_{k+1} \tilde{x}_{k+1 \mid k} \tilde{x}_{k+1 \mid k}^{T}+\epsilon_{k+1}^{-1} \hat{x}_{k+1 \mid k} \hat{x}_{k+1 \mid k}^{T},
\end{aligned}
$$

with $\epsilon_{k+1}$ being a positive scalar. Then, the term $\mathbb{E}\left\{x_{k+1} x_{k+1}^{T}\right\}$ can be calculated as

$$
\begin{aligned}
& \mathbb{E}\left\{x_{k+1} x_{k+1}^{T}\right\} \\
= & \mathbb{E}\left\{\left(\tilde{x}_{k+1 \mid k}+\hat{x}_{k+1 \mid k}\right)\left(\tilde{x}_{k+1 \mid k}+\hat{x}_{k+1 \mid k}\right)^{T}\right\} \\
\leq & \left(1+\epsilon_{k+1}\right) P_{k+1 \mid k}+\left(1+\epsilon_{k+1}^{-1}\right) \hat{x}_{k+1 \mid k} \hat{x}_{k+1 \mid k}^{T} .
\end{aligned}
$$

Thus, it follows from (15) and (25) that

$$
\begin{aligned}
& P_{k+1 \mid k+1} \\
\leq & \left(\mathbb{I}-K_{k+1} \bar{\Lambda}_{k+1} C_{k+1}\right) P_{k+1 \mid k}\left(\mathbb{I}-K_{k+1} \bar{\Lambda}_{k+1} C_{k+1}\right)^{T} \\
& +K_{k+1}\left[\check{\Lambda}_{k+1} \circ\left(C_{k+1} \Psi_{k+1} C_{k+1}^{T}\right)\right] K_{k+1}^{T} \\
& +K_{k+1} R_{k+1} K_{k+1}^{T},
\end{aligned}
$$

where $\Psi_{k+1}=\left(1+\epsilon_{k+1}\right) P_{k+1 \mid k}+\left(1+\epsilon_{k+1}^{-1}\right) \hat{x}_{k+1 \mid k} \hat{x}_{k+1 \mid k}^{T}$. Therefore, it is easy to see that $P_{k+1 \mid k+1} \leq \Xi_{k+1 \mid k+1}$.

Finally, we are in a position to show that the upper bound $\Xi_{k+1 \mid k+1}$ can be minimized by using the estimator parameter $K_{k+1}$ in (22). Note that $\Xi_{k+1 \mid k+1}$ in (20) can be rewritten as

$$
\begin{aligned}
\Xi_{k+1 \mid k+1}= & \Xi_{k+1 \mid k}-K_{k+1} \bar{\Lambda}_{k+1} C_{k+1} \Xi_{k+1 \mid k}-\Xi_{k+1 \mid k} \\
& \times C_{k+1}^{T} \bar{\Lambda}_{k+1}^{T} K_{k+1}^{T}+K_{k+1} S_{k+1} K_{k+1}^{T}
\end{aligned}
$$

where $S_{k+1}$ is defined as in (23). Substituting $K_{k+1}=$ $\sum_{i=1}^{N}\left(\Phi_{1, i}^{T} K_{i, k+1} \Phi_{2, i}\right)$ into (27), the trace of the matrix $\Xi_{k+1 \mid k+1}$ can be calculated as follows:

$$
\begin{aligned}
& \operatorname{tr}\left(\Xi_{k+1 \mid k+1}\right) \\
= & \operatorname{tr}\left[\Xi_{k+1 \mid k}-\sum_{i=1}^{N}\left(\Phi_{1, i}^{T} K_{i, k+1} \Phi_{2, i}\right) \bar{\Lambda}_{k+1} C_{k+1} \Xi_{k+1 \mid k}\right. \\
& -\Xi_{k+1 \mid k} C_{k+1}^{T} \bar{\Lambda}_{k+1}^{T} \sum_{i=1}^{N}\left(\Phi_{1, i}^{T} K_{i, k+1} \Phi_{2, i}\right)^{T} \\
& \left.+\sum_{i=1}^{N}\left(\Phi_{1, i}^{T} K_{i, k+1} \Phi_{2, i}\right) S_{k+1}\left(\Phi_{1, i}^{T} K_{i, k+1} \Phi_{2, i}\right)^{T}\right],(28)
\end{aligned}
$$

where $\Phi_{1, i}$ and $\Phi_{2, i}$ are defined as in (23). It is worth mentioning that, when deriving (28), we have used the following fact

$\operatorname{tr}\left[\left(\Phi_{1, i}^{T} K_{i, k+1} \Phi_{2, i}\right) S_{k+1}\left(\Phi_{1, j}^{T} K_{j, k+1} \Phi_{2, j}\right)^{T}\right]=0$,

for $i \neq j$. According to Lemma 3, taking the partial derivative of the trace of $\Xi_{k+1 \mid k+1}$ with respect to $K_{i, k+1}$ and letting the derivative be zero, we obtain

$$
\begin{aligned}
\frac{\partial \operatorname{tr}\left(\Xi_{k+1 \mid k+1}\right)}{\partial K_{i, k+1}}= & -2 \Phi_{1, i} \Xi_{k+1 \mid k} C_{k+1}^{T} \bar{\Lambda}_{k+1}^{T} \Phi_{2, i}^{T} \\
& +2 \Phi_{1, i} \Phi_{1, i}^{T} K_{i, k+1} \Phi_{2, i} S_{k+1} \Phi_{2, i}^{T} \\
= & 0 .
\end{aligned}
$$

Noting $S_{k+1}>0$, then we can see that $\Phi_{2, i} S_{k+1} \Phi_{2, i}^{T}$ is invertible. Through the algebraic manipulations, it 
follows from (29) and $\Phi_{1, i} \Phi_{1, i}^{T}=I_{n \times n}$ that the estimator parameter can be given by

$K_{i, k+1}=\Phi_{1, i} \Xi_{k+1 \mid k} C_{k+1}^{T} \bar{\Lambda}_{k+1}^{T} \Phi_{2, i}^{T}\left(\Phi_{2, i} S_{k+1} \Phi_{2, i}^{T}\right)^{-1}$,

which completes the proof of this theorem.

Remark 4 Note that considerable research effort has been made on the state estimation problems for complex networks. However, almost all the state estimation schemes can only be applied for the time-invariant complex networks concerning the asymptotical behavior of the estimation error dynamics. It is worth mentioning that the newly developed state estimation approach can be used for an array of time-varying stochastic complex networks by evaluating the transient behavior of the estimation error covariance over a specified time horizon. Moreover, the optimization of the upper bound of the estimation error covariance has been conducted by properly designing the estimator parameters which can be seen as another distinguishing feature. On the other hand, when the boundednedss analysis of the estimation error becomes a concern, some additional constraints on the system parameters can be introduced as in [16] to ensure the boundedness of the estimation errors over the whole time horizon, which constitutes one of future research topics.

Remark 5 During the design process of the state estimation algorithm, the scalars $\epsilon_{k+1}$ and $\gamma_{k}$ have been introduced with hope to increase the freedom of the proposed estimation algorithm. It is worth pointing out that the parameter $\gamma_{k}$ affects the solvability of the equations (19) and (20). In the implementation, the value of $\gamma_{k}$ could be given a prior and adjusted to help enhance the solvability and feasibility of the developed estimation scheme. Moreover, it should be kept in mind that the computational burden and difficulties grow when the scale of a network system is large.

Remark 6 For the stochastic time-varying complex network model (1)-(2) under consideration, there are five main aspects which complicate the design of the state estimator, i.e., the nonlinear function $f(\cdot)$, the coupling structure, the time-varying nature, the probabilistic missing measurements as well as the stochastic disturbances. In Theorem 3, sufficient conditions, which include all of the information on these five aspects, are established for the existence of a state estimator to satisfy the locally minimized error-variance performance requirement. The corresponding solvability conditions for the desired state estimator gains are expressed in terms of the feasibility of a series of Riccati-like matrix difference equations that are of a recursive form suitable for online computation.

\section{A Numerical Example}

In this section, a simulation example is presented to illustrate the usefulness of the established state estimation scheme.

Consider a time-varying stochastic complex network (1)(2) with four nodes. The coupling configuration matrix is assumed to be $W=\left(\omega_{i, j}\right)_{N \times N}$ with

$\omega_{i, j}=\left\{\begin{array}{cc}-0.3, & i=j \\ 0.1, & i \neq j\end{array}\right.$ and the inner-coupling matrix is given by $\Gamma=0.2 I_{2} \cdot \varpi_{i, k}$ $(i=1,2,3,4)$ are zero-mean Gaussian white noises with covariances $Q_{1, k}=0.05, Q_{2, k}=0.03, Q_{3, k}=0.04$ and $Q_{4, k}=0.02, \nu_{i, k}$ are zero-mean Gaussian white noises with covariances $R_{1, k}=0.03, R_{2, k}=0.02, R_{3, k}=0.04$ and $R_{4, k}=0.06$. The matrices $B_{i, k}$ and $C_{i, k}$ are taken as

$$
\begin{aligned}
& B_{1, k}=\left[\begin{array}{c}
0.05-0.01 \sin (3 k) \\
0.02
\end{array}\right], \quad B_{2, k}=\left[\begin{array}{c}
-0.03 \\
0.12
\end{array}\right], \\
& B_{3, k}=\left[\begin{array}{l}
0.02 \\
0.06
\end{array}\right], \quad B_{4, k}=\left[\begin{array}{c}
0.04 \\
-0.01
\end{array}\right], \\
& C_{1, k}=\left[\begin{array}{ll}
0.90 & 0.25
\end{array}\right], \quad C_{2, k}=\left[\begin{array}{ll}
0.95 & 0.65
\end{array}\right], \\
& C_{3, k}=\left[\begin{array}{ll}
0.90 & 0.35
\end{array}\right], \quad C_{4, k}=\left[\begin{array}{ll}
0.85 & 0.95
\end{array}\right] .
\end{aligned}
$$

The nonlinear function $f\left(x_{i, k}\right)$ is chosen as

$f\left(x_{i, k}\right)=\left[\begin{array}{c}0.8 x_{i, k}^{(1)}+\sin \left(x_{i, k}^{(1)} x_{i, k}^{(2)}\right) \\ 1.5 x_{i, k}^{(2)}-\sin \left(x_{i, k}^{(1)} x_{i, k}^{(2)}\right)\end{array}\right]$,

where $x_{i, k}=\left[x_{i, k}^{(1)} x_{i, k}^{(2)}\right]^{T}$ is the system state. Assume that the probability mass functions for $\lambda_{i, k}(i=1,2,3,4)$ are:

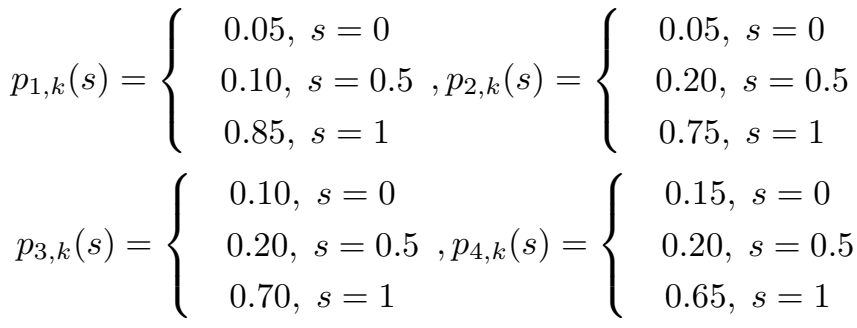

Accordingly, the expectation and variance of $\lambda_{1, k}$ can be easily calculated as $\mu_{1, k}=0.9$ and $\sigma_{1, k}^{2}=0.065$. Similarly, we have $\mu_{2, k}=0.85, \mu_{3, k}=0.8, \mu_{4, k}=0.75$, $\sigma_{2, k}^{2}=0.0775, \sigma_{3, k}^{2}=0.11$ and $\sigma_{4, k}^{2}=0.1375$. The other parameters are chosen as $\bar{x}_{1,0}=\left[\begin{array}{lll}1.80 & -0.25\end{array}\right]^{T}$, $\bar{x}_{2,0}=[1.75-0.20]^{T}, \bar{x}_{3,0}=\left[\begin{array}{lll}1.75 & -0.25\end{array}\right]^{T}$ and $\bar{x}_{4,0}=[1.80-0.25]^{T}, \Xi_{1,0 \mid 0}=20 I_{2}, \Xi_{2,0 \mid 0}=25 I_{2}$, $\Xi_{3,0 \mid 0}=30 I_{2}, \Xi_{4,0 \mid 0}=35 I_{2}, \epsilon_{k+1}=0.2, \gamma_{k}=0.03$, $L_{1, k}=\operatorname{diag}\{0.02,0.03\}, L_{2, k}=\operatorname{diag}\{0.01,0.03\}$, $L_{3, k}=\operatorname{diag}\{0.03,0.01\}$ and $L_{4, k}=\operatorname{diag}\{0.01,0.05\}$.

By using Theorem 3, the estimator parameters can be obtained recursively. The simulations are given in Figs. 4-4, where the trajectories of the actual states $x_{i, k}^{(j)}$ and their estimates $\hat{x}_{i, k}^{(j)}(j=1,2)$ are plotted. From the simulation, we can see that the designed state estimators have a satisfactory tracking performance in the simultaneous presence of missing measurements and stochastic disturbances, which further confirm the feasibility and effectiveness of the state estimation scheme developed in this paper. 


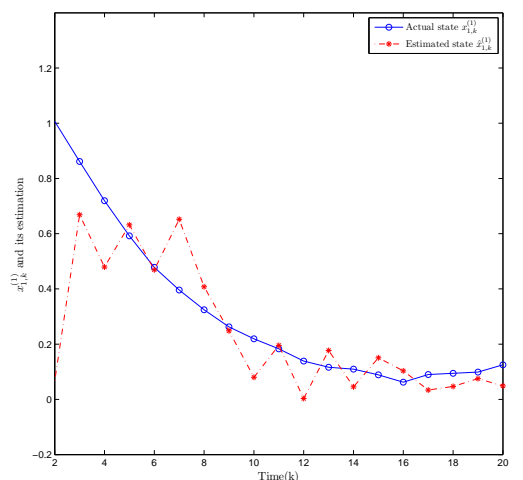

Fig. 1. Actual state $x_{1, k}^{(1)}$ and its estimation $\hat{x}_{1, k}^{(1)}$

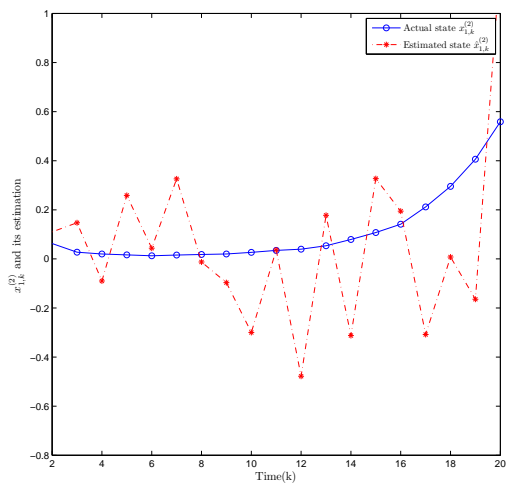

Fig. 2. Actual state $x_{1, k}^{(2)}$ and its estimation $\hat{x}_{1, k}^{(2)}$

\section{Conclusions}

In this paper, we have made one of the first few attempts to investigate the recursive state estimation problem for an array of discrete time-varying stochastic complex networks with missing measurements and random disturbances. A recursive state estimator has been constructed in which the missing probability has been introduced to compensate the effect from the missing measurements to the estimation performance. Further research topics include the extension of the developed results to the state estimation problem for a more general class of timevarying complex networks with event-triggering transmission mechanism and/or network-induced phenomena in the near future.

\section{References}

[1] Barabási, A. L., \& Albert, R. (1999). Emergence of scaling in random networks. Science, 286(5439), 509-512.

[2] Basin, M. V., Shi, P., \& Calderon-Alvarez, D. (2011). Joint state filtering and parameter estimation for linear stochastic time-delay systems. Signal Processing, 91(4), 782-792.

[3] Caballero-Águila, R., Hermoso-Carazo, A., Jiménez-López, J. D., Linares-Pérez, J., \& Nakamori, S. (2010). Signal estimation with multiple delayed sensors using covariance information. Digital Signal Processing, 20(2), 528-540.

[4] Calafiore, G. (2005). Reliable localization using set-valued nonlinear filters. IEEE Transactions on Systems, Man, and Cybernetics-Part A: Systems and Humans, 35(2), 189-197.

[5] Ding, D., Wang, Z., Shen, B., \& Dong, H. (2015). Envelopeconstrained $H_{\infty}$ filtering with fading measurements and

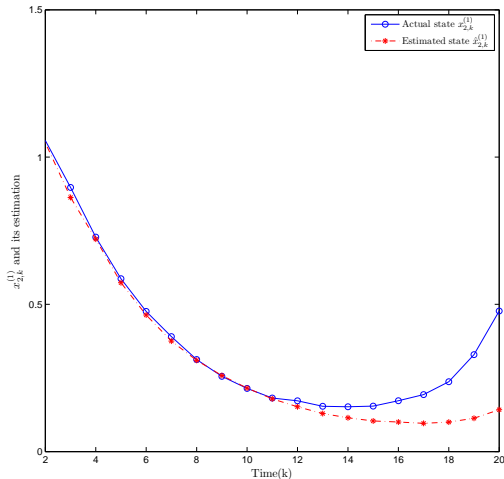

Fig. 3. Actual state $x_{2, k}^{(1)}$ and its estimation $\hat{x}_{2, k}^{(1)}$

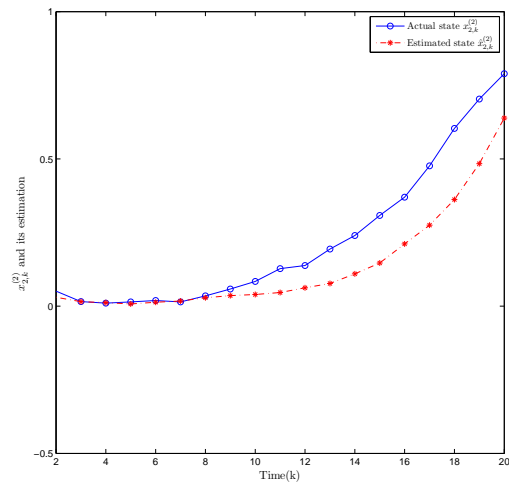

Fig. 4. Actual state $x_{2, k}^{(2)}$ and its estimation $\hat{x}_{2, k}^{(2)}$

randomly occurring nonlinearities: the finite horizon case. Automatica, 55, 37-45.

[6] Ding, D., Wang, Z., Lam, J., \& Shen, B. (2015). FiniteHorizon $H_{\infty}$ control for discrete time-varying systems with randomly occurring nonlinearities and fading measurements. IEEE Transactions on Automatic Control, 60(9), 2488-2493.

[7] Dong, H., Wang, Z., Ding, S. X., \& Gao, H. (2014). Finitehorizon estimation of randomly occurring faults for a class of nonlinear time-varying systems. Automatica, 50(12), 31823189.

[8] Gao, H., Chen, T., \& Lam, J. (2008). A new delay system approach to network-based control. Automatica, 44(1), 3952.

[9] Haughton, D. A., \& Heydt, G. T. (2013). A linear state estimation formulation for smart distribution systems. IEEE Transactions on Power Systems, 28(2), 1187-1195.

[10] Horn, R. A., \& Johnson, C. R. (1991). Topic in Matrix Analysis, New York: Cambridge University Press.

[11] Hounkpevi, F. O., \& Yaz, E. (2007). Robust minimum variance linear state estimators for multiple sensors with different failure rates. Automatica, 43(7), 1274-1280.

[12] Hu, J., Wang, Z., Gao, H., \& Stergioulas, L. K. (2012). Extended Kalman filtering with stochastic nonlinearities and multiple missing measurements. Automatica, 48(9), 20072015.

[13] Hu, J., Wang, Z., Shen, B., \& Gao, H. (2013). Quantized recursive filtering for a class of nonlinear systems with multiplicative noises and missing measurements. International Journal of Control, 86(4), 650-663.

[14] Jawadekar, A., Paraskar, S., Jadhav, S., \& Dhole, G. (2014). Artificial neural network-based induction motor 


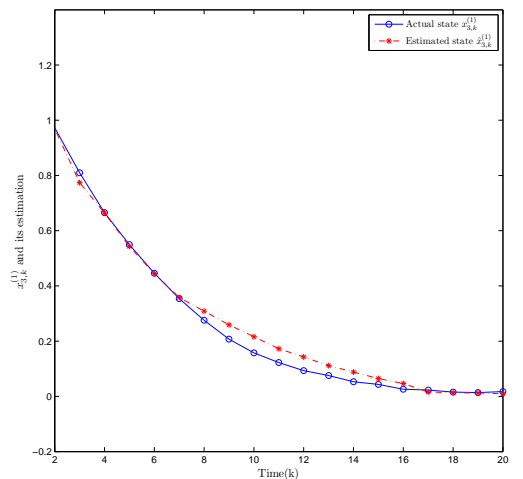

Fig. 5. Actual state $x_{3, k}^{(1)}$ and its estimation $\hat{x}_{3, k}^{(1)}$

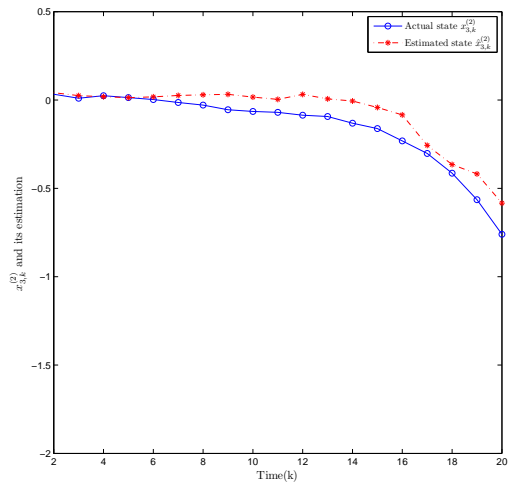

Fig. 6. Actual state $x_{3, k}^{(2)}$ and its estimation $\hat{x}_{3, k}^{(2)}$

fault classifier using continuous wavelet transform. Systems Science and Control Engineering: An Open Access Journal, 2(1), 684-690.

[15] Romero-Cadaval, E., Spagnuolo, G., Franquelo, L. G., Ramos-Paja, C. A., Suntio, T., \& Xiao, W. M. (2013). Gridconnected photovoltaic generation plants components and operation. IEEE Industrial Electronics Magazine, 7(3), 6-20.

[16] Kluge, S., Reif, K., \& Brokate, M. (2010). Stochastic stability of the extended Kalman filter with intermittent observations. IEEE Transactions on Automatic Control, 55(2), 514-518.

[17] Liang, J., Wang, Z., \& Liu, X. (2009). State estimation for coupled uncertain stochastic networks with missing measurements and time-varying delays: the discrete-time case. IEEE Transactions on Neural Networks, 20(5), 781793.

[18] Niu, Y., \& Ho, D. W. C. (2010). Design of sliding mode control subject to packet losses. IEEE Transactions on Automatic Control, 55(11), 2623-2628.

[19] Wang, T., Gao, H., \& Qiu, J. A combined adaptive neural network and nonlinear model predictive control for multirate networked industrial process control. IEEE Transactions on Neural Networks and Learning Systems, DOI: 10.1109/TNNLS.2015.2411671.

[20] Porfiri, M., \& Bernardo, M. (2008). Criteria for global pinning-controllability of complex networks. Automatica, 44(12), 3100-3106.

[21] Shen, B., Wang, Z., \& Liu, X. (2011). Bounded $H_{\infty}$ synchronization and state estimation for discrete timevarying stochastic complex networks over a finite horizon. IEEE Transactions on Neural Networks, 22(1), 145-157.

[22] Shen, B., Wang, Z., Ding, D., \& Shu, H. (2013). $H_{\infty}$ state estimation for complex networks with uncertain inner

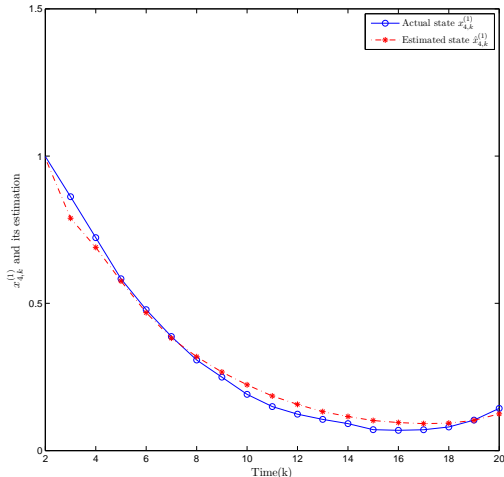

Fig. 7. Actual state $x_{4, k}^{(1)}$ and its estimation $\hat{x}_{4, k}^{(1)}$

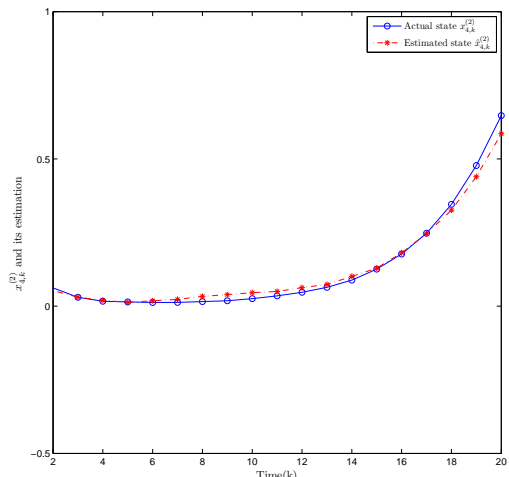

Fig. 8. Actual state $x_{4, k}^{(2)}$ and its estimation $\hat{x}_{4, k}^{(2)}$

coupling and incomplete measurements. IEEE Transactions on Neural Networks and Learning Systems, 24(12), 20272037.

[23] Strogatz, S. H., \& Watts, D. J. (1998). Collective dynamics of 'small-world' networks. Nature, 393(6684), 440-442.

[24] Wei, G., Wang, Z., \& Shu, H. (2009). Robust filtering with stochastic nonlinearities and multiple missing measurements. Automatica, 45(3), 836-841.

[25] Wang, Y., Xie, L., \& de Souza, C. E. (1992). Robust control of a class of uncertain nonlinear systems. Systems \& Control Letters, 19(2), 139-149.

[26] Wang, Z., Wang, Y., \& Liu, Y. (2010). Global synchronization for discrete-time stochastic complex networks with randomly occurred nonlinearities and mixed time delays. IEEE Transactions on Neural Networks, 21(1), 11-25.

[27] Xiong, K., Wei, C., \& Liu, L. (2010). Robust extended Kalman filtering for nonlinear systems with stochastic uncertainties. IEEE Transactions on Systems, Man, and Cybernetics-Part A: Systems and Humans, 40(2), 399-405.

[28] Yang, X., Cao, J., \& Lu, J. (2012). Stochastic synchronization of complex networks with nonidentical nodes via hybrid adaptive and impulsive control. IEEE Transactions on Circuits and Systems-I, Regular Paper, 59(2), 371-384.

[29] Yang, X., Cao, J., \& Lu, J. (2013). Synchronization of randomly coupled neural networks with markovian jumping and time-delay. IEEE Transactions on Circuits and Systems I-Regular Papers, 60(2), 363-376. 